\title{
The effect of non-uniform current distribution on transport current loss in stacked high- $T_{c}$ superconductor tapes
}

\author{
Seyong Choi ${ }^{1}$, Wansoo $\mathrm{Nah}^{2^{*}}$, Jinho $\mathrm{Joo}^{2}$, Kyung-Woo Ryu ${ }^{3}$, \\ Byoung-Seob Lee ${ }^{1}$, Jang-Hee Yoon ${ }^{1}$, Jung-Woo Ok ${ }^{1}$, Jin Yong Park ${ }^{1}$, Mi-Sook Won ${ }^{{ }^{*}}$ \\ ${ }^{1}$ Busan Center, Korea Basic Science Institute, Busan 609-735, Korea \\ ${ }^{2}$ Sngkyunkwan University, Gyeonggi 440-746, Korea \\ ${ }^{3}$ Chonnam National University, Gwangju 500-757, Korea
}

Received 26 March 2012; accepted 16 May 2012

\begin{abstract}
The influence of current distribution on the transport current loss in vertically stacked high- $T_{c}$ superconductor (HTS) tapes was evaluated. AC loss was analyzed as a function of current distribution by introducing a current distribution parameter through a numerical method (finite element analysis). AC loss under non-uniform current distribution is always higher than that for a uniformly distributed transport current in a conductor. Although the effect of non-uniformity is relatively insignificant in low transport current, AC loss increases substantially in high transport current regions as non-uniformity is enlarged. The results verify that non-uniform current distribution causes extra loss by examining the cross-sectional view of current densities in stacked conductor.
\end{abstract}

\section{INTRODUCTION}

It is well known that the current carrying capabilities of HTS equipment can be increased via the use of a multi-stacked or stranded conductor using HTS tapes. The non-uniform current distribution in such conductors may result in the degradation of overall performance and stability [1]. It has well reported that the main cause of non-uniform current distribution arises from joint resistance at the terminal, unbalanced inductance between layers, different impedance according to the winding pitch, etc. [1]-[6]. The determination of the critical current of a HTS apparatus and measurement of AC loss is a complicated issue [7]-[9]. In the past, a number of attempts have been made to reduce AC loss by making the current distribution as even as possible, but it is necessary to systematically analyze the effects of non-uniform current distribution on AC loss.

In this paper, we report an examination the effect of current distribution on AC loss in stacked Bi-2223/Ag tapes. In our previous studies, a numerical method was proposed to predict AC transport current losses in vertically stacked HTS tapes. The proposed numerical method was confirmed with experimental results and was found to be in quite good agreement with each other

\footnotetext{
* Corresponding author: wsnah@skku.edu, mswon@kbsi.re.kr
}

TABLE I

SPECIFICATIONS OF HTS TAPE FOR STACKED CONDUCTOR.

\begin{tabular}{lc}
\hline Composition & $(\mathrm{Bi}, \mathrm{Pb}) 2223 / \mathrm{Ag}$ \\
Size & $3.1 \times 0.17 \mathrm{~mm}$ \\
Filamentary region & $3.0 \times 0.11 \mathrm{~mm}$ \\
Number of filaments & 55 \\
Twist & Not twisted \\
Critical current at $77 \mathrm{~K}$ & $75-80 \mathrm{~A}$ \\
Index $n$ & 20 \\
\hline
\end{tabular}

[11]-[12]. The numerical methods to predict AC loss in stacked Bi-2223/Ag tapes for different current distribution are described here. AC transport current losses were analyzed by introducing a current distribution parameter. Using the current distribution parameter, AC transport current losses are explained and discussed with numerical results as well as drawings of current density distribution in stacked Bi-2223/Ag tapes.

\section{NUMERICAL ANALYSIS}

\subsection{Stacked HTS Model}

An Ag-alloy sheathed Bi-2223 superconducting tape was considered in this work. The detailed specifications of Bi-2223/Ag tapes are listed in Table. I. Fig. 1 shows a schematic diagram of stacked conductor. As can be seen in Fig. 1, the stacked conductor consists of three Bi-2223/Ag tapes in a manner of one tape is overlaid on another. Each tape is numbered as Tape 1, Tape 2, and Tape 3, from top to bottom. Also, the current flowing into each tape is defined by $I_{i}(i=1,2,3)$, which corresponds to the current at Tape 1 , Tape 2, and Tape 3, respectively. The critical current of each HTS tape and a model conductor were measured at 77 $\mathrm{K}$ by the standard four-probe method. The critical currents of each tape were almost the same, about 75-80 A and the measured critical currents of the model conductor was 193 A. The critical current of stacked conductor showed significant degradation considering the simple summation of critical current in each tape. It is, however, well known 


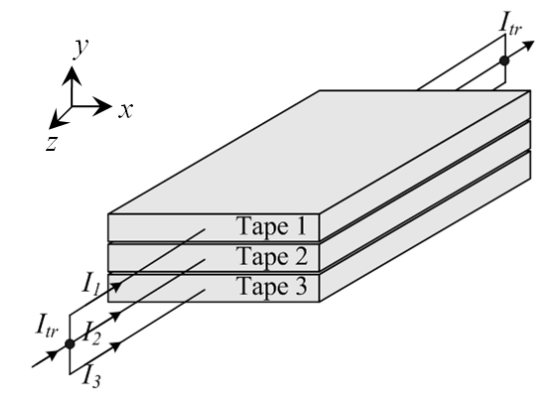

Fig. 1. Schematic diagram of a vertically stacked conductor.

that a magnetic field generated by a neighboring tape has significantly been affected the critical current of a stacked conductor if the tapes are stacked closely [9]-[12].

\subsection{The Current Distribution Parameter}

In our analysis, a sinusoidal transport current $I_{t r}(t)=I_{0}$. $\sin (w t)$ was applied to a model conductor, as shown in Fig. 1. At the beginning of the conductor, the applied transport current $I_{t r}$ was branched off and flowed to each tape $I_{i}$, which had the relation: $I_{t r}=\sum I_{i}(i=1,2$, and 3$)$. To examine the effect of current non-uniformity on AC loss, a current distribution parameter $\gamma$, defined as the ratio of $I_{1}$ to $I_{2}$ or $I_{3}$ to $I_{2}$, was introduced although this parameter cannot be used for practical situation. When current distribution parameter is 1 , it is assumed that all of the current flowing is completely identical. If $\gamma$ is below or above 1 , this indicates that at least the amplitudes of the current in some tapes are different, i.e. non-uniformly distributed current in the conductor.

\subsection{Numerical Analysis}

The numerical approach based on finite element method was used to calculate AC loss. The numerical analysis was implemented with an $\boldsymbol{A}-\boldsymbol{V}$ formulation, as was used in previous studies [11]-[12]. As shown in Fig. 1, electromagnetic quantities of a stacked conductor should be only limited in terms of the $z$-component. Therefore, a two-dimensional analysis is adequate for a model conductor. The governing equation is defined as:

$$
\nabla \cdot\left(\nabla A_{z}\right)=-\mu_{0} \sigma(|B|, \theta)\left(\nabla V+\frac{\partial A_{z}}{\partial t}\right)
$$

where, $A_{z}$ is the magnetic vector potential, $V$ is electric scalar potential, $\mu_{0}$ is magnetic permeability of vacuum, and $t$ is the time. The field-dependent, non-linear superconducting property $(\sigma)$ should be considered for exact loss estimation. This can be expressed as a function of the magnetic field and its orientation as in (2), which can be obtained from the power law and ohm's law based on the sets of the measured critical current and index number as a function of various external magnetic fields:

$$
\sigma(|B|, \theta)=\frac{|E|^{\frac{1-n|B|, \theta)}{n(|B|, \theta)}}}{E_{c}^{\frac{1}{n(|B|, \theta)}}} \cdot J_{c}(|B|, \theta)
$$

where, $B$ is the magnetic field density, $\theta$ is the incident angle of $B, E$ is the electric field, and $J_{c}$ and $n$ denote the critical current density and index number of the Bi-2223/Ag tape. From the simultaneous calculation with (1) and (2), AC transport current loss can be obtained from an integration of the current density and electric field over the cross-section of the conductor and one period of time.

\section{RESUltS AND DISCUSSION}

Fig. 2 depicts numerically estimated AC transport current losses $\left(Q_{t r}\right)$ with respect to the normalized current of a stacked conductor. The normalized current in the figure means the transport current $\left(I_{t r}\right)$ divided by the critical current of the stacked conductor $\left(I_{c}\right)$. In a previous study, it was confirmed that the proposed numerical method for accurately predicting AC loss in a stacked conductor was in good agreement with the experimental results [9]-[12]. From the validity of the presented method, the influence of current distribution on AC loss was analyzed. Fig. 2 indicates the effect of current distribution parameters on AC transport current loss. As explained above, the currents at each tape were uniformly or non-uniformly distributed for various $\gamma$ values. The AC loss calculations were performed for same transport current by changing the value of $\gamma$ from 0.5 to 1.5. As shown in Fig. 2, the value of AC loss is always the smallest in the case of $\gamma$ $=1.0$, which is the uniformly distributed current in the conductor. The evaluated results for non-uniform current distribution generally showed higher losses than $\gamma_{-}=1.0$. This fact suggests that the AC losses increase according to the non-uniformity of the current distribution. This is due to the fact that non-uniform current distribution causes extra AC loss, which was excessive compared to the uniform current distribution. For the case of a low $I_{t r}$, the effect of current distribution on loss is insignificant, regardless of the value of $\gamma$. However, it is also found from Fig. 2 that AC losses are highly dependent on $\gamma$ and deviations according to $\gamma$ become enlarged substantially, especially in a high $I_{t r}$ region around the $I_{c}$. To see the distinct effect of current distribution parameter on AC losses in more detail, we depicted several AC loss results as the ratio of $\gamma \neq 1$ to $\gamma=1$ in Fig. 3 and 4 for the sake a comparison.

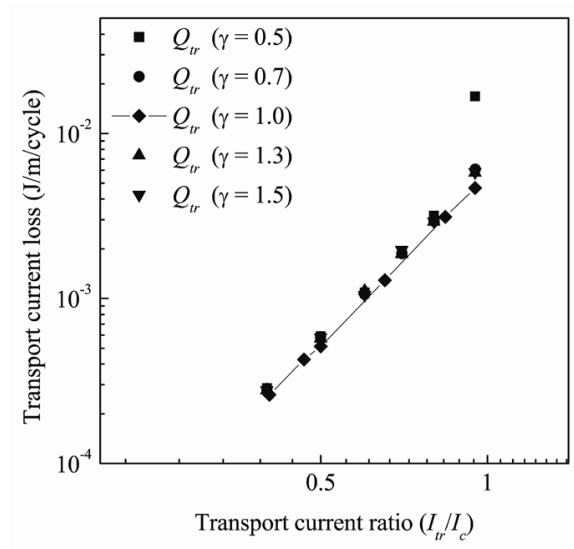

Fig. 2. AC transport current losses for various current distribution parameters. 


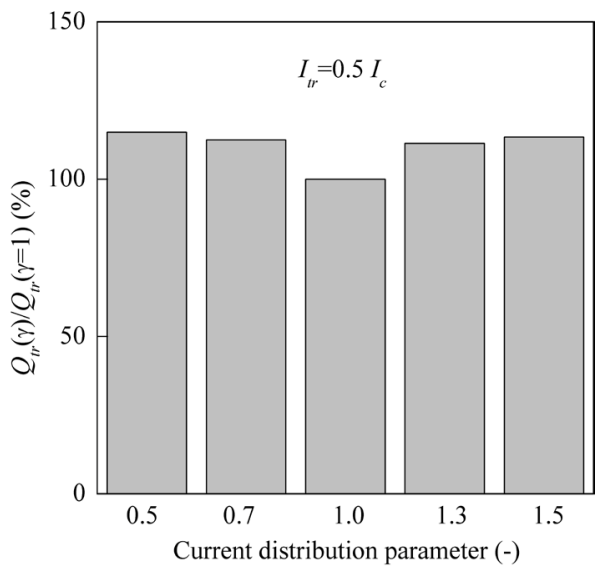

Fig. 3. AC loss ratio for different current distribution parameter normalized by the loss of uniform current distribution $(\gamma=1)$ when $I_{t r}=0.5 I_{c}$.

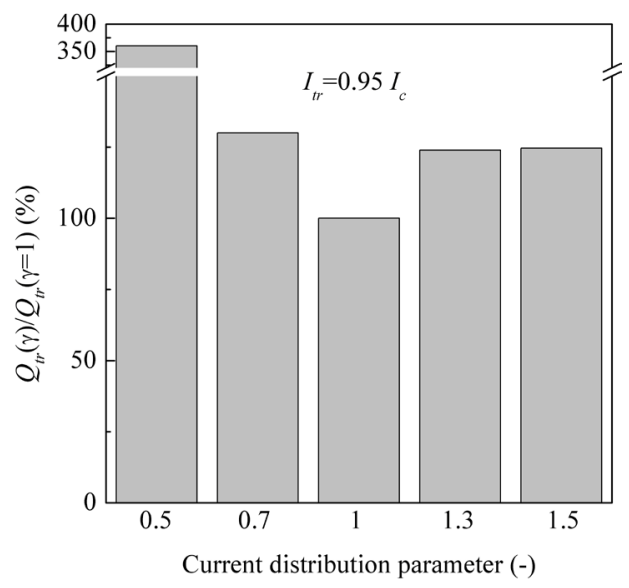

Fig. 4. AC loss ratio for different current distribution parameter normalized by the loss of uniform current distribution $(\gamma=1)$ when $I_{t r}=0.95 I_{c}$.

AC losses in Fig. 2 were replotted for specific transport currents as in Fig. 3 and Fig. 4. The figures show the increment of AC loss at $\gamma \neq 1$ divided by the loss when $\gamma=1$. In Fig. 3, AC losses are compared with each other according to $\gamma$ when $I_{t r}=0.5 I_{c}$. One can be seen in this figure all of the losses in non-uniform current distribution are slightly higher than $\gamma=1$. On the contrary, AC loss is much higher than $\gamma=1$ as non-uniformity increases for a high transport current $\left(I_{t r}=0.95 I_{c}\right)$ as in Fig. 4 .

The effect of non-uniformity for different values of $\gamma$ can be understood by inspecting the current flowing $I_{i}$ at each tape. For an extreme parameter such as $\gamma=0.5$ or 1.5 , a transport current of $0.5 I_{c}$ is far below $I_{c}$, the currents in each tape are also below its $I_{c}$. The differences between $I_{i}$ are negligible in the low $I_{t r}$ region, although $\gamma$ is not uniform. This fact leads to an insignificant effect of the $\gamma$ in case of a low $I_{t r}$. In Fig.4, the transport current of $I_{t r}=0.95 I_{c}$ is still below the critical current of conductor but the current in some of the tape is well over its $I_{c}$. For instance, for $\gamma=0.5$, the current at Tape $2\left(I_{2}=92 \mathrm{~A}\right)$ is much higher than its $I_{c}$ (75-80 A). This kind of non-uniformity causes a
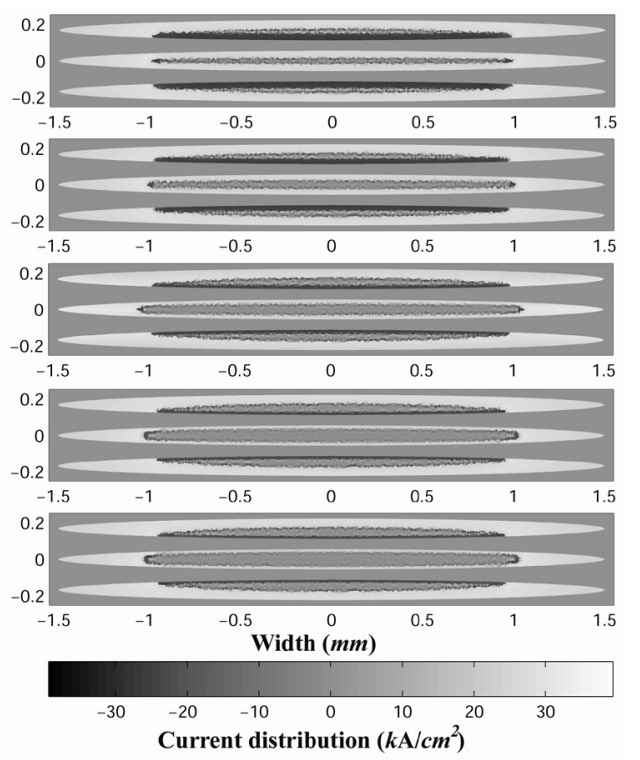

Fig. 5. The illustrations of current density when $I_{t r}=0.5 I_{c}$, $\omega t=\pi / 2$. The current distribution parameters are $0.5,0.7$, $1.0,1.3$, and 1.5 from top to bottom, respectively.
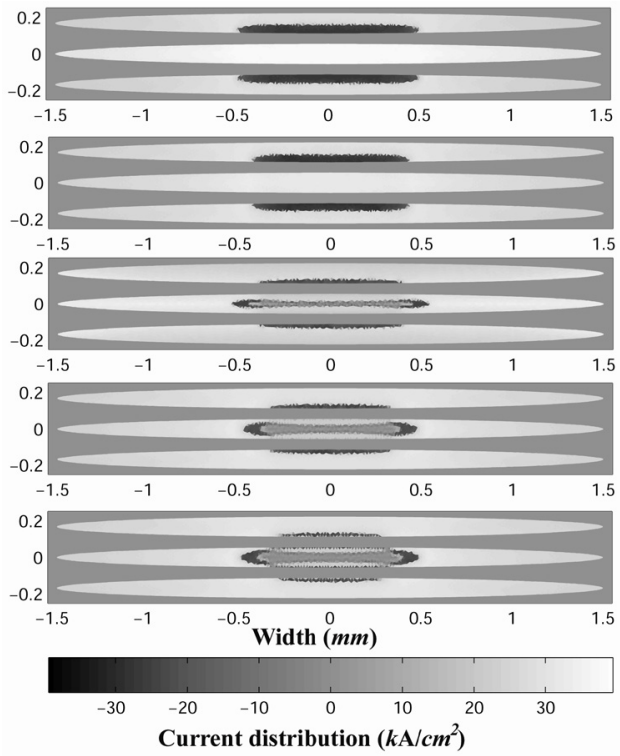

Fig. 6. The illustrations of current density when $I_{t r}=0.95 I_{c}$, $\omega t=\pi / 2$. The current distribution parameters are $0.5,0.7$, $1.0,1.3$, and 1.5 from top to bottom, respectively.

considerable AC loss increment in the high $I_{t r}$ region. Above the critical current, flux flow begins and flux flow loss is supposed to be more dominant mechanism than flux creep loss in the AC loss. Those results suggest that the reduction of $\mathrm{AC}$ loss can be achieved by making the current as equal as possible even though the current is non-uniformly distributed.

Fig. 5 and Fig. 6 illustrates the distribution in current density across the cross-section of the stacked conductor at $\omega t=\pi / 2$, which is related to the instant peak magnitude of the applied current. From top to bottom, each figure corresponds to $\gamma=0.5,0.7,1.0,1.3$, and 1.5 , respectively. 
In each cross-section of conductor, the one from top, middle, and bottom tapes also indicate Tape 1, 2, and 3 . The transport current in Fig. 5 and 6 are individually shown for $0.5 I_{c}$ and $0.95 I_{c}$. The drawings of current density at a low $I_{t r}$, as in Fig. 5, are quite similar to one another regardless of the values of $\gamma$. The findings indicate that there is only a miniscule difference in current density even in extreme cases $(\gamma=0.5$ or 1.5$)$.

It is reasonable that the value of $\gamma$ is negligibly influenced on AC loss in low $I_{t r}$. While at a high $I_{t r}$ of $0.95 I_{c}$ noticeable difference can be seen in Fig. 6 and the current density depends much more on various $\gamma$ values than in Fig. 5. This could be a plausible answer for why the effect of $\gamma$ value on $\mathrm{AC}$ transport loss increases as non-uniformity severely goes from $\gamma=1.5$ to 0.5 . Especially, this fact can be clearly explained in Fig. $6(\gamma=0.5)$ that no current-free region is observed in Tape 2 because the current density is fully penetrated in the tape cross-section. The AC loss is more significantly affected by non-uniform current distribution at high $I_{t r}$.

\section{CONCLUSION}

AC transport current losses for stacked conductor consisting of $\mathrm{Bi}-2223 / \mathrm{Ag}$ tapes were investigated. A numerical calculation for AC loss estimation was performed to examine the effect of current distribution on $\mathrm{AC}$ transport current loss. The findings indicate that $\mathrm{AC}$ loss when the transport current is uniform in each tape always shows a lower behavior than non-uniform current distribution, which causes an increase in AC loss. At the low transport current region, AC loss was not significantly affected by the current distribution. While for a high transport current, AC loss increased considerably in comparison with uniform current distribution and the effect of current distribution parameter on AC loss increased with increasing non-uniformity. The cross-sectional view of current density from the numerical calculation also verified that the uniformity of current distribution turned out to significantly affect AC loss, especially in the high transport current region.

\section{ACKNOWLEDGMENT}

This work was supported by the KBSI Grant No. D31300.

\section{REFERENCES}

[1] Vladimir R. Romanovskii, "Current Degradation of Multi-Strand Superconducting Cable Caused by Non-Ideal Inductive Coupling Between Its Components", IEEE Trans. Appl. Supercond., Vol.12, No.1, pp.1578-1581, 2002

[2] M. Daumling, "A Model for the current distribution and ac losses in superconducting power cables", Cryogenics 39, pp.759-766, 1999.

[3] Jin-Hong Joo, Seog-Whan Kim, Jeonwook Cho, Joon Han Bae, Hae Jong Kim, Ki Chul Seong and Jung Pyo Hong, "Pitch Calculation of 4-layer HTS Power Transmission Cable for Balanced Sharing Current", Journal of Korea Institute of Applied Superconductivity and Cryogenics, Vol.5, No.1, pp.35-39, 2003.

[4] Duck-Kweon Bae, Hyoungku Kang, Min-Cheol Ahn, Yeong-Sik Kim, Kyung-Yong Yoon, Yong-Soo Yoon, Jun-Han Bae, Tae-Kuk Ko, "Current Sharing of Parallel Connected Bi-2223 High- $T_{c}$ Superconducting paths", Journal of Korea Institute of Applied Superconductivity and Cryogenics, Vol.6, No.2, pp.20-24, 2004.

[5] Y. H. Ma, Kyung-Woo Ryu, K. B. Park, Il-Sung Oh, "Effect of Conductor's Arrangement and Current Direction on AC Loss Characteristics of a Fault Current Limiting Coil", Journal of Korea Institute of Applied Superconductivity and Cryogenics, Vol.7, No.3, pp.17-20, 2005

[6] T. Mito, K. Takahata, A. Iwamoto, R. Maekawa, N. Yanagi, T. Satow, O. Motojima, and J. Uamamoto, "Extra AC losses for a CICC coil due to the nonuniform current distribution in the cable", Cryogenics, vol. 38, pp. 551-558, 1998.

[7] Naoyuki Amemiya, "Experimental and numerical approaches for AC loss estimation in high $\mathrm{T}_{\mathrm{c}}$ superconductors", Journal of Korea Institute of Applied Superconductivity and Cryogenics, Vol.6, No.2, pp.1-6, 2004.

[8] Naoyuki Amemiya, "A Review on AC Loss Characteristics of High $\mathrm{T}_{\mathrm{c}}$ Superconductors", Journal of Korea Institute of Applied Superconductivity and Cryogenics, Vol.8, No.2, pp.1-7, 2006.

[9] K. Ryu, S. D. Hwang, S. W. Kim, and S. Hahn, "Influence of tape's critical currents and current distributions on AC loss measurement in a multi-tape conductor," IEEE Trans. Appl. Supercond., Vol.15, No.2, pp.1611-1614, 2005.

[10] Seyong Choi, Su-Jeong Suh, Wansoo Nah, Yong Ho Ma, Kyung-Woo Ryu, Jung Ho Kim, Jinho Joo, Myung-Hwan Sohn, and Young-Kil Kwon, "Influence of Non-Uniform Current Distribution on AC Transport Current Loss in Bi-2223/Ag Tapes", IEEE Trans. Appl. Supercond., Vol.16, No.2, pp.154-157, 2006.

[11] Seyong Choi, Wansoo Nah, Jung Ho Kim, Jinho Joo, Young-Ho Jo, Kyung-Woo Ryu, "AC transport current loss of vertically stacked Bi-2223/Ag conductors", Physica C 412-424, pp.1158-1162, 2004.

[12] Seyong Choi, Wansoo Nah, Jung Ho Kim, Jinho Joo, Young-Ho Jo, Kyung-Woo Ryu, "AC transport current loss of horizontally attached Bi-2223/Ag tapes", IEEE Trans. Appl. Supercond., Vol.14, pp.1894-1897, 2004 